\title{
Application of Micro-Tech stents in malignant carinal stenosis
}

\author{
JIN-MU NIU, JIE ZHANG，XIAO-JIAN QIU，JUAN WANG，YING-HUA PEI，YU-LING WANG and TING WANG
}

\author{
Department of Respiration, Beijing Tian Tan Hospital, Capital Medical University, \\ Dongcheng, Beijing 100050, P.R. China
}

Received July 19, 2018; Accepted February 1, 2019

DOI: $10.3892 / 01.2019 .10051$

\begin{abstract}
Malignant carinal stenosis is a disease process that is not always suitable for treatment with a Y-shaped stent. When one of the main bronchi is completely obstructed and cannot be recanalized, or its distal lung tissue has lost function, inserting a Y-shaped stent is infeasible. In this complex condition, a cone-shaped stent is selected to maintain the patency of the trachea and the other main bronchus. The efficacy of the bare cone-shaped Micro-Tech stent to treat malignant carinal stenosis was evaluated in the current study. The medical records of 47 patients with malignant carinal stenosis who underwent Micro-Tech stent placement between January 2004 and October 2017 in Beijing Tian Tan Hospital (Beijing, China) were analyzed retrospectively. A total of 47 bare Micro-Tech stents (28 Y-shaped and 19 cone-shaped) were successfully inserted in the patients. Following stent placement, immediate satisfactory results were achieved in all patients, especially an improvement of dyspnea (100\%), cough (81.1\%) and stridor (100\%). The Karnofsky scores were significantly increased $(\mathrm{P}<0.001)$, and the American Thoracic Society Dyspnea Index values were significantly decreased $(\mathrm{P}<0.001)$. Following a median of 88 days, initial bronchoscopic interventions were performed for tumor overgrowth (84.6\%), stent fracture (7.7\%) and granulation tissue $(7.7 \%)$. Restenting was performed in two patients due to tumor progression $(n=1)$ and stent fracture $(n=1)$. Removal of a Y-shaped stent was attempted in one patient, but failed, as the stent had become partly embedded in the airway mucosa. In conclusion, cone-shaped Micro-Tech stent placement maintained tracheal-unilateral main bronchus patency and benefitted patients with malignant carinal stenosis in whom Y-shaped stents were not suitable for insertion.
\end{abstract}

\section{Introduction}

Primary and metastatic malignancies can occur in the carinal region, leading to carinal stenosis $(1,2)$. The majority of

Correspondence to: Dr Jie Zhang, Department of Respiration, Beijing Tian Tan Hospital, Capital Medical University, 6 Tiantanxili, Dongcheng, Beijing, 100050, P.R. China

E-mail: zhangj_tt@163.com

Key words: malignant carinal stenosis, cone-shaped stent, Y-shaped stent, initial experience, bronchoscopic intervention patients with malignant carinal stenosis are diagnosed in the advanced stages of the disease and cannot undergo surgical resection (3). In these cases, bronchoscopic interventions including stent placements may be the only palliative option for maintaining lumen patency (4). Bronchoscopic interventions are advantageous as they are less invasive and may be suitable for critically ill patients (5). Malignant carinal stenosis can occasionally be accompanied by airway distortion, making stent selection and placement difficult. Y-shaped silicon stents are traditionally used to treat malignant carinal stenosis $(6,7)$. However, their disadvantages include poor shape-adaptability, difficult placement and secretion retention $(8,9)$. In addition, when one of the main bronchi is completely obstructed and cannot be recanalized, or its distal lung tissue has lost function, inserting a Y-shaped stent is not possible (4). Micro-Tech stents (Micro-Tech Co., Ltd., Nanjing, China) are self-expanding metal stents available in different shapes and are individually customizable. The current study described the use of Micro-Tech stents in malignant carinal stenosis. To the best of our knowledge, this is the first study to evaluate the feasibility of using the bare cone-shaped metal stent to treat malignant carinal stenosis for which Y-shaped stents are not suitable.

\section{Materials and methods}

Patients. A total of 47 patients (38 males and 9 females; median age, 59 years; age range, $23-83$ years) with malignant carinal stenosis who underwent Micro-Tech stent placement in Beijing Tian Tan Hospital (Beijing, China) between January 2004 and October 2017 were enrolled. All patients could not undergo surgical treatment due to advanced disease stage and 13 of them experienced postoperative tumor recurrence. Data including patient demographics, medical histories, interventional therapies administered before stenting, complications during stenting and outcomes following stenting were analyzed retrospectively. The current study was approved by The Ethical Review Committee of Beijing Tiantan Hospital, Capital Medical University (approval ID: JS2013-007-02). All patients signed an informed consent form before the insertion of the Micro-Tech stent.

Preparation prior to stenting. Prior to stent placement, patients underwent examinations including electrocardiograms and blood tests including routine blood counts, coagulation tests, liver and kidney function tests and electrolyte levels. Computed tomography scans of the chest, including coronal and sagittal 
reconstruction, and bronchoscopy were performed to assess the extent, type and degree of stenosis, which provided a reliable basis for stent selection and size customization $(10,11)$. Preoperative and preanesthetic evaluations were conducted by interventional pulmonologists and anesthetists, respectively. During the stent placements, dynamic electrocardiograms, blood pressure and oxygen saturation levels $\left(\mathrm{SaO}_{2}\right)$ were monitored. If general anesthesia was administered, arterial blood gases were also analyzed.

Stent selection and deployment. Uncovered Y-shaped and cone-shaped Micro-Tech stents were used in the current study. The stents and stent delivery systems were manufactured by Micro-Tech Co. Ltd.

$Y$-shaped stents. The Y-shaped stent has three bifurcations comprising one tracheal and two bronchial limbs. The length and diameter of the limbs and the angle between the two bronchial limbs can be individually customized in various combinations. The tracheal limb lengths of the Y-shaped stents used in the current study ranged between 30 and $60 \mathrm{~mm}$, and the diameters ranged between 14 and $20 \mathrm{~mm}$. The bronchial limb lengths ranged between 10 and $45 \mathrm{~mm}$, and diameters ranged between 8 and $14 \mathrm{~mm}$. The angles between the two bronchial limbs ranged between 60 and $90^{\circ}$. The Y-shaped stent was preferred when the two sides of the main bronchus remained functional despite carinal involvement causing lower tracheal and bilateral proximal main bronchial obstruction (Fig. 1A).

The Y-shaped stent was loaded into an introducer sheath. Prior to stent placement, a rigid bronchoscope (RB) was inserted. Through the RB, a 0.035-inch guide wire (Boston Scientific Corporation, Marlborough, MA, USA) was inserted in the left or right main bronchus using a bronchoscope. The bronchoscope was subsequently withdrawn and the delivery system was advanced into the carina with the corresponding bronchial limb of the stent passing over the guide wire (Fig. 1B). The bronchial limbs were exposed following initial retraction of the introducer sheath. Under direct visualization through an ultra-thin flexible bronchoscope (outer diameter, $2.8 \mathrm{~mm}$ ), the stent bronchial limbs bound by threads were inserted into the relevant main bronchus (Fig. 1C). The bronchial limbs were released by pulling the threads through the ring-pull in the delivery system. The tracheal limb was released by further withdrawal of the introducer sheath, thus completing the stent placement (Fig. 1D). The Y-shaped stent can also be inserted orally. With the patients under total intravenous anesthesia and remaining spontaneous respiration, both the guide wire and delivery system were inserted orally. The release process of the stent was the same as aforementioned. In the present study, 12 out of 28 Y-shaped stents were inserted orally.

Cone-shaped stents. The cone-shaped stent is a special straight stent constructed according to the authors' specifications. The opposite ends of the stent have different diameters, allowing a smooth transition between the large and small ends. The proximal (tracheal) end should be positioned in the trachea while the distal (bronchial) end should be positioned in the main bronchus, thus maintaining tracheal-unilateral main bronchus patency. The diameter of the two ends and the length of the stent can be individually customized. The lengths of the cone-shaped stent used in this study ranged between 50 and $80 \mathrm{~mm}$. The stent diameters ranged between 10 and $18 \mathrm{~mm}$, and the difference between the diameters of the two ends varied between 2 and $8 \mathrm{~mm}$. Once placed in trachea-left (or right) main bronchus, the stent blocked the opening of the other main bronchus (Fig. 2B). Although airflow could pass through the stent mesh, further bronchoscopic interventions would not be possible. Only when a unilateral main bronchus is obstructed and cannot be recanalized (Fig. 2A), or its distal lung tissue is nonfunctional, can a cone-shaped stent insertion be considered (4).

Cone-shaped stent insertions are simple and almost identical to the process for traditional straight Micro-Tech stents. Prior to stent insertion, the depth of the delivery system to be inserted into the airway was measured with a bronchoscope. The distance from the distal end of the bronchoscope to the proximal end of the airway was measured. A guide wire was subsequently inserted into the functional main bronchus through the working channel of the bronchoscope. Following removal of the bronchoscope, the delivery system was inserted into the airway, passing over the guide wire until it reached the measured depth. The stent was then released by withdrawing the introducer sheath. Following the release of the stent, the bronchoscope was re-inserted to confirm proper stent placement and expansion. If necessary, biopsy forceps could be used to adjust the stent.

Efficacy evaluation. Procedural efficacy was evaluated according to respiratory symptom improvement and according to the differences in the American Thoracic Society Dyspnea Index (12) and Karnofsky score (13) prior to and following stent placement.

Follow-up. Patients underwent bronchoscopy within one week following stenting to assess early complications ( $\leq 1$ week). Bronchoscopy was subsequently performed every 1-3 months, or for worsening dyspnea, and late complications ( $>1$ week) were recorded. When necessary, bronchoscopic interventions were performed. The follow-up ended upon the start of this study (January 2018), at the patient's last visit to our hospital, or upon the patient's mortality.

Statistical analysis. Statistical data analysis was performed using SPSS version 22.0 (IBM Corp., Armok, NY, USA). The American Thoracic Society Dyspnea Index and Karnofsky score data are expressed as the mean \pm standard deviation. Comparisons of the American Thoracic Society Dyspnea Index and Karnofsky score before and following stent placement were made using paired-sample t-tests. $\mathrm{P}<0.05$ was considered to indicate a statistically significant difference.

\section{Results}

Characteristics of the patients. Forty-seven patients (38 males and 9 females) with a median age of 59 years (ranging between 23 and 83 years) were enrolled. The patients' symptoms included dyspnea $(93.6 \% ; 44 / 47)$, cough $(78.7 \% ; 37 / 47)$, hemoptysis $(48.9 \% ; 23 / 47)$, dysphagia $(6.4 \% ; 3 / 47)$, chest pain $(4.3 \% ; 2 / 47)$, hoarseness $(4.3 \% ; 2 / 47)$ and stridor $(2.1 \% ; 1 / 47)$. 

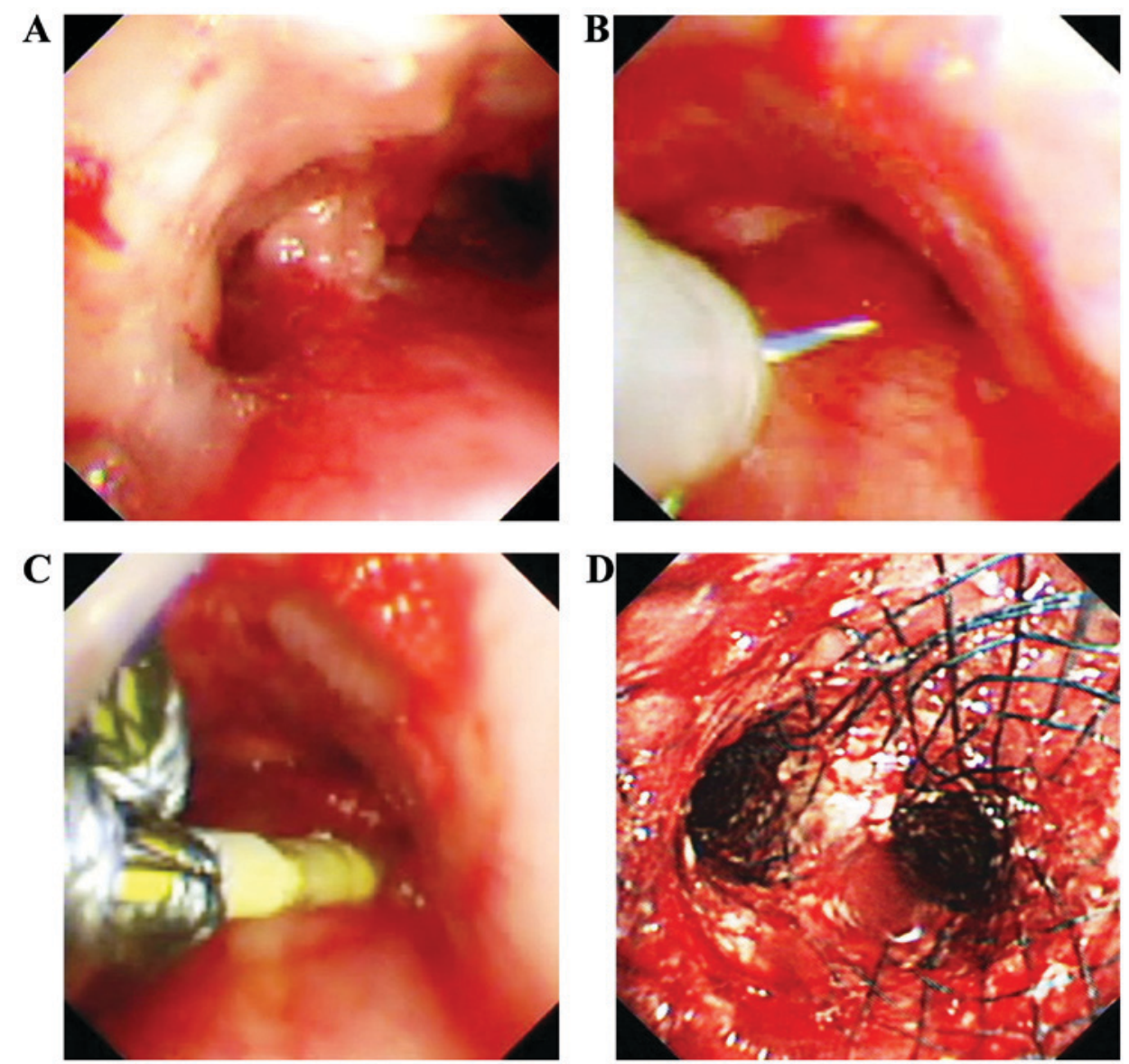

Figure 1. Endoscopic images of a malignant carinal stenosis that is suitable for the insertion of a Y-shaped stent. (A) Malignant carinal stenosis involving the trachea and bilateral main bronchus. Y-shaped stent insertion is generally required. (B) The Y-shaped Micro-Tech stent delivery system is inserted into the carina with the bronchial limb passing over a guide wire. (C) The bronchial limbs of a Y-shaped Micro-Tech stent are inserted into the relevant main bronchus under direct visualization using an ultra-thin flexible bronchoscope. (D) The placement of the entire Y-shaped Micro-Tech stent is completed.

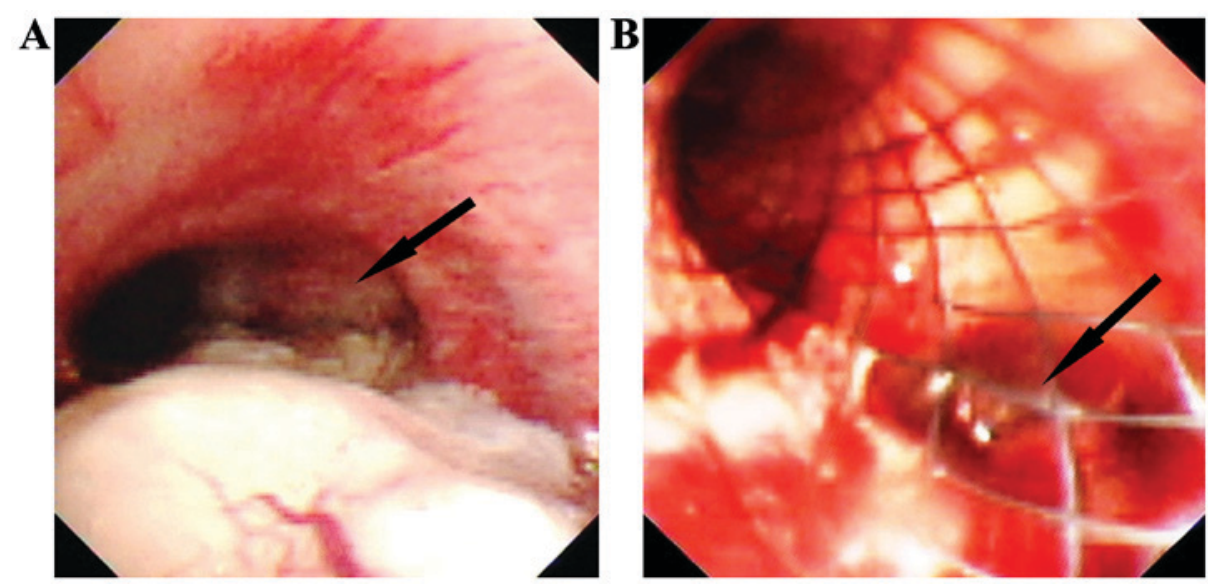

Figure 2. Endoscopic images of a malignant carinal stenosis that is suitable for the insertion of a cone-shaped stent. (A) Specific malignant carinal stenosis. The right bronchus is completely obstructed by the tumor and cannot be recanalized (arrow), which makes inserting a Y-shaped stent not possible. (B) A cone-shaped Micro-Tech stent is inserted in the trachea-left main bronchus. The arrow points to the opening of the right main bronchus that is blocked by the stent.

Of all the patients, $27.7 \%(13 / 47)$ had atelectasis and $19.1 \%$ (9/47) had obstructive pneumonia. The lesion etiologies included primary $(87.2 \% ; 41 / 47)$ and metastatic $(12.8 \% ; 6 / 47)$ malignancies (Table I). Squamous cell and esophageal carcinomas were the most common primary and metastatic tumors, respectively. Twenty-nine patients had treatment histories that included surgery $(44.8 \% ; 13 / 29)$, chemotherapy $(51.7 \% ; 15 / 29)$, bronchoscopic intervention $(31.0 \% ; 9 / 29)$ and radiotherapy (44.8\%; 13/29). Prior to stent placement, 34 patients underwent bronchoscopic interventions including argon plasma coagulation $\left(47.1 \%\right.$; 16/34), carbon dioxide $\left(\mathrm{CO}_{2}\right)$ cryotherapy $(23.5 \%$; $8 / 34)$, high-frequency electric knife $(67.6 \% ; 23 / 34)$, snare $(17.6 \% ; 6 / 34)$, balloon dilatation $(5.9 \% ; 2 / 34)$ and laser therapy $(17.6 \% ; 6 / 34)$ to reduce airway obstruction. 
Table I. Lesion etiology.

A, Primary tumors

\begin{tabular}{lcc}
\hline Lesion etiology & n (patients) & \% of 47 patients \\
\hline Squamous cell carcinoma & 26 & 55.3 \\
Adenocarcinoma & 7 & 14.9 \\
Adenoid cystic carcinoma & 5 & 10.6 \\
Mucoepidermoid carcinoma & 2 & 4.3 \\
Undifferentiated carcinoma & 1 & 2.1 \\
\hline
\end{tabular}

B, Metastatic tumors

\begin{tabular}{lcc}
\hline Lesion etiology & $\mathrm{n}$ (patients) & $\%$ of 47 patients \\
\hline Esophageal cancer & 4 & 8.5 \\
Malignant lymphoma & 1 & 2.1 \\
Liver cancer & 1 & 2.1 \\
\hline
\end{tabular}

Anesthesia and ventilation methods. Anesthesia and ventilation methods during stent placement are presented in Table II. In $12.5 \%(1 / 8)$ of the patients with an endotracheal tube (ETT), part of the cone-shaped stent entered into the ETT following release. When withdrawing the ETT, the stent migrated and required removal. Ultimately, the stent was successfully reinserted through a laryngeal mask (LAM). Obvious $\mathrm{CO}_{2}$ retention occurred in $26.3 \%(5 / 19)$ of patients in whom an $\mathrm{RB}$ was used. Following stent placement, RBs were replaced with LAMs to promote $\mathrm{CO}_{2}$ removal. Among patients with spontaneous ventilation under total intravenous anesthesia (TIVA-SV), 8.3\% (1/12) experienced a decrease in $\mathrm{SaO}_{2}$ due to glossoptosis and nasopharyngeal airways were required.

Outcomes. Forty-seven bare stents including 28 Y-shaped and 19 cone-shaped stents were successfully inserted in all 47 patients. Reversible bleeding $(19.1 \%$; 9/47) and glottic edema $(4.3 \% ; 2 / 47)$ were the most common procedure-associated complications. No serious complications were observed.

Intervention efficacy. All patients achieved immediate symptomatic improvement, especially in dyspnea (100\%; 44/44), cough $(81.1 \%$; 30/37) and stridor $(100 \% ; 1 / 1)$. The Karnofsky scores were significantly increased from $62.77 \pm 12.105$ to $80.64 \pm 8.445(\mathrm{t}=-17.769 ; \mathrm{P}<0.001)$ and the American Thoracic Society Dyspnea Index scores were significantly decreased from $3.06 \pm 0.895$ to $1.60 \pm 0.712(\mathrm{t}=16.224 ; \mathrm{P}<0.001)$.

Follow-up. A total of 42 patients were assessed for early complications (Table III). Mucosal necrosis was the most common early complication and was removed using biopsy forceps. The early occurrence of granulation tissue was mild and did not cause airway obstruction. One cone-shaped stent migrated distally and was adjusted successfully using biopsy forceps.

Twenty patients were followed-up long-term, with a median length of 116 days (range, 16-1,537 days). Among the late complications (Table IV), three Y-shaped stents fractured and the duration of time prior to the fractures was 32, 93 and 497 days, respectively. Despite fracture, two out of the three stents did not compromise airway support. No migrations, perforations, fistulas or stent-associated mortalities were observed. Thirteen of the 20 patients underwent at least one (range, 1-6) bronchoscopic intervention due to aggravation of dyspnea caused by tumor overgrowth or stent-associated complications. Following each interventional treatment, dyspnea was improved to varying degrees. Following a median time of 88 days (range, 35-388 days), initial bronchoscopic interventions were performed due to tumor overgrowth $(84.6 \% ; 11 / 13)$, stent fracture $(7.7 \% ; 1 / 13)$ and granulation tissue $(7.7 \%$; $1 / 13$; data not shown). Restenting was performed in two patients as stenosis progressed over the existing stent $(n=1)$ and a fractured stent led to compromised airway support $(n=1)$. Eighty-eight days following insertion, a Y-shaped stent removal was attempted in a patient with malignant lymphoma as the stenosis improved following tumor-specific chemotherapy. As the stent was partly embedded in the airway mucosa, the removal attempt failed.

\section{Discussion}

At present, treatments for malignant carinal stenosis are challenging (14-16). Various malignances can occur in the carinal region including the lower trachea and the proximal end of the bilateral main bronchus (1). Dyspnea, cough and hemoptysis are commonbut nonspecific respiratory symptoms (17). Since the majority of patients are already in the advanced stages of the disease at the time of diagnosis, only a number of patients have the opportunity for surgical treatment (3). Carinal surgery is technically difficult and often accompanied by high morbidity and mortality rates (18-20). It is reported that mortality rates following carinal resection and reconstruction range from $29-40 \%$ (3) and surgery may be avoided at the expense of an improved prognosis (14). Yamamoto et al (14) recommended that if possible, surgery should still be performed in selected patients. A study by Shin et al (3) included 30 patients with locally advanced non-small cell lung cancer involving the carinal and/or trachea underwent carinal resection and reconstruction. The surgical candidacy was evaluated according to guidelines based on pulmonary function, general conditions and co-morbidity of the patient, as well as the extent of the tumor invasion, nodal stage and alternative non-surgical modalities. For patients who cannot be treated surgically, bronchoscopic intervention is an alternative palliative treatment that can effectively remove intraluminal tumors $(21,22)$. When the structure of the airway wall is destroyed by tumor infiltration, or stenosis is caused by compression from an external mass or lymph node, stent insertion should be considered (23). In the current study, certain patients could not undergo surgical treatment due to advanced disease stage or tumor recurrence following surgery. Although the majority of patients (72.3\%) underwent bronchoscopic intervention prior to stent placement, stent insertion was still required due to mixed obstructions.

Y-shaped stents are used to maintain patency of the lower trachea and the proximal end of the bilateral main bronchus. Y-shaped silicone stents are traditionally used and 
Table II. Anesthesia and ventilation methods.

\begin{tabular}{lcccc}
\hline & \multicolumn{4}{c}{$\mathrm{n}$ (cone-shaped stent, Y-shaped stent) } \\
\cline { 2 - 4 } Type of anesthesia & LAM & ETT & RB & Tubeless \\
\hline General anesthesia & $8(8,0)$ & $7(7,0)$ & $19(3,16)$ & $0(0,0)$ \\
Local anesthesia + conscious sedation & $0(0,0)$ & $1(1,0)$ & $0(0,0)$ & $0(0,0)$ \\
TIVA-SV & $0(0,0)$ & $0(0,0)$ & $0(0,0)$ & $12(0,12)$ \\
\hline
\end{tabular}

LAM, laryngeal mask; ETT, endotracheal tube; RB, rigid bronchoscopy; TIVA-SV, spontaneous ventilation under total intravenous anesthesia.

Table III. Early complications.

\begin{tabular}{lccc}
\hline Complication & $\begin{array}{c}\text { Cone-shaped stent } \\
\mathrm{n} \text { (\% of 17 stents) }\end{array}$ & $\begin{array}{c}\text { Y-shaped stent } \\
\mathrm{n} \text { (\% of 25 stents) }\end{array}$ & $\mathrm{n}$ (\% of 42 stents) \\
\hline Mucosal necrosis & $11(64.7)$ & $19(76.0)$ & $30(71.4)$ \\
Increased secretions & $10(58.8)$ & $18(72.0)$ & $28(66.7)$ \\
Granulation tissue & $1(5.9)$ & $1(4.0)$ & $2(4.8)$ \\
Mucositis & $0(0.0)$ & $2(8.0)$ & $2(4.8)$ \\
Glottis edema & $0(0.0)$ & $1(4.0)$ & $1(2.4)$ \\
Migration & $1(5.9)$ & $0(0.0)$ & $1(2.4)$ \\
Bleeding & $1(5.9)$ & $0(0.0)$ & $1(2.4)$ \\
\hline
\end{tabular}

Table IV. Late complications.

\begin{tabular}{lccc}
\hline Complication & $\begin{array}{c}\text { Cone-shaped stent } \\
\mathrm{n} \text { (\% of 7 stents) }\end{array}$ & $\begin{array}{c}\text { Y-shaped stent } \\
\mathrm{n} \text { (\% of 13 stents) }\end{array}$ & $\mathrm{n}(\%$ of 20 stents) \\
\hline Tumor overgrowth & $7(100.0)$ & $9(69.2)$ & $16(80.0)$ \\
Phlegm retention & $4(57.1)$ & $5(38.5)$ & $9(45.0)$ \\
Granulation tissue & $2(28.6)$ & $6(46.2)$ & $8(40.0)$ \\
Fracture & $0(0.0)$ & $3(23.1)$ & $3(15.0)$ \\
Bleeding & $1(14.3)$ & $1(7.7)$ & $2(10.0)$ \\
Scarring & $0(0.0)$ & $1(7.7)$ & $1(5.0)$ \\
Epithelialization & $0(0.0)$ & $1(7.7)$ & $1(5.0)$ \\
\hline
\end{tabular}

have favorable safety and tolerance profiles $(6,7)$. However, they are difficult to insert, especially for complex carinal stenosis with distorted airways. Once deployed, they can interfere with mucociliary clearance, resulting in secretion retention (23). Compared to silicone stents, self-expanding Y-shaped metal stents are relatively easy to insert and have improved expansion force (16). Following insertion, they can gradually expand to a predetermined shape that matches the contour of the tracheobronchial tree and requires less routing care (8). Studies on the treatment of malignant carinal stenosis with Y-shaped metal stents have been reported (15,24-26). Madan et al (15) reported one of the largest studies on Y-shaped metal stents. The authors used the fully covered metal Y-shaped stent, which has the advantage of preventing tumor ingrowth. In the present study, the Y-shaped stents used were uncovered. The advantage of the uncovered stent is that it does not easily migrate and has little effect on mucociliary clearance (23). The efficacy and safety of Y-shaped covered stents and Y-shaped uncovered stents requires further comparison. In certain patients with long segments of tracheal obstruction in addition to carinal stenosis, combination airway stenting using tracheal and Y-shaped stents may be required (27).

Malignant carinal stenosis can occasionally be accompanied by loss of function of one of the main bronchi, making insertion of a Y-shaped stent infeasible (4). In the current study, the most common reasons for loss of function of a main bronchus are complete luminal obstruction that precludes recanalization, or surgical removal of the distal lung tissue. The current study demonstrated the feasibility of inserting cone-shaped stents to maintain tracheal and unilateral main bronchus patency in malignant carinal stenosis. 
Compared with silicone stent, inserting a Micro-Tech stent is relatively easy (28). In the current study, all stents were successfully inserted without serious procedure-associated complications and immediate and satisfactory effects were achieved. The choice of anesthesia and ventilation methods during stent insertion should depend on the stent type, location of lesions and severity of airway lesions (29). In the current study, the majority of stents were inserted through an artificial airway with the patient under general anesthesia for the following reasons: i) Patients were unable to tolerate local anesthesia due to severe airway obstruction; ii) bronchoscopic intervention was performed to remove intraluminal masses before stenting, and the operation time was lengthy; iii) procedure-associated complications could occur and would need to be managed; and iv) patients required general anesthesia due to discomfort with local anesthesia. ETTs can provide safe and reliable ventilation access, but they occupy part of the airway space and are not conducive to stent release in the upper and middle trachea (29). LAM is a supraglottic airway device and provides excellent visibility of the glottis and subglottis; thus, it could be regarded as a reliable alternative for airway management during interventional bronchoscopic procedures, particularly when they are located near the glottis or in the upper trachea (30). When using an $\mathrm{RB}$, the presence of mobile teeth and the extent of neck mobility should be considered (29). In addition, high-frequency ventilation through $\mathrm{RBs}$ reduces exhalation time and may lead to $\mathrm{CO}_{2}$ retention (31). TIVA-SV is an alternative anesthetic method (32). In this current study, the insertion of the Y-shaped stent with the patients under TIVA-SV was always successful.

In the current study, complications following stent placement could be effectively managed endoscopically when necessary. Mucosal necrosis was the most common early complication. This was usually observed in the areas of airway lesions or at the ends of the stent. This may be due to the greater contact pressure between the mucosa and the stent in these areas. Stent migration was only observed as an early complication as opposed to a late complication. Following long-term implantation, stents were embedded in the malignant or granulation tissues, or epithelialization occurred. These were also the reasons why metal stents were difficult to remove. Metal stents can fracture following placement, and the free stent wire could protrude into the bronchial lumen or damage the surrounding tissue. In addition, the fractured stent may lose the ability to support the airway and should be replaced with a new one or covered by inserting a new stent over the fracture (33). Tumor overgrowth was the most common reason for repeated bronchoscopic interventions following stent insertion (34). When the disease progresses and the stenosis is beyond the scope of the original stent, restenting remains an effective treatment method (33).

Removal of metal stents is challenging; potential risks include stent fracture, mucosal tear, hemorrhage, restenosis, pneumothorax and mortality (35). The longer the stent is present in the airway, the more difficult it is to remove (36). Due to the limited survival rate of patients with malignant carinal stenosis, few stent removals are considered. The main indication for stent removal is disease improvement (37) or severe stent-associated complications (38). In the current study, a patient with malignant lymphoma exhibited disease improvement following tumor-specific chemotherapy, and stent removal was attempted but failed. Therefore, if possible, removable stents may be more appropriate for patients with untreated malignancies that are sensitive to radiotherapy and chemotherapy $(9,39)$.

This study had a number of limitations. First, the details of anesthesia and airway management performed by the anesthetist were not presented as they were unavailable. Second, the patients attended follow-up unequally. In this study, the majority of patients were referred from other hospitals and eventually lost to follow-up. Therefore, patients' survival time was rarely recorded. Previous studies have indicated that the median survival time of patients with malignant carinal stenosis following stenting varied between 46 and 181 days, and even if patients with malignant carinal stenosis receive bronchoscopic interventions including stenting, their prognosis remains poor $(2,7,16)$.

In conclusion, the current study initially described the insertion of uncovered cone-shaped stents and demonstrated the feasibility of their use for maintaining tracheal-unilateral main bronchus patency in malignant carinal stenosis. The uncovered Micro-Tech stent placement (including Y-shaped and cone-shaped types) is a safe and effective palliative treatment for patients with malignant carinal stenosis.

\section{Acknowledgements}

The authors thank Dr Min Xu and Dr Chen-Yang Zhang (both from the Department of Respiration, Beijing Tian Tan Hospital, Capital Medical University, Beijing, China) for their assistance in collecting data.

\section{Funding}

This study was supported by the Capital Health Development Research Project (grant no. 2016-2-2048).

\section{Availability of data and materials}

The datasets used and analyzed during the current study are available from the corresponding author on reasonable request.

\section{Authors' contributions}

JZ and JMN designed the study. JMN collected and analyzed the data, and drafted the manuscript. JZ, XJQ, JW, YHP, YLW, and TW performed the stent placements or bronchoscopic interventions, and revised the manuscript. All authors have read and approved the final manuscript.

\section{Ethics approval and consent to participate}

The study was approved by the Ethical Review Committee of Beijing Tiantan Hospital, Capital Medical University (Beijing, China; approval ID: JS2013-007-02). All patients signed an informed consent form before the insertion of the Micro-Tech stent.

\section{Patient consent for publication}

Not applicable. 


\section{Competing interests}

The authors declare that they have no competing interests.

\section{References}

1. Puchalski J and Musani AI: Tracheobronchial stenosis: Causes and advances in management. Clin Chest Med 34: 557-567, 2013

2. Tsukioka T, Takahama M, Nakajima R, Kimura M, Tei K and Yamamoto R: Sequential stenting for extensive malignant airway stenosis. Ann Thorac Cardiovasc Surg 21: 114-118, 2015.

3. Shin S, Park JS, Shim YM, Kim HJ and Kim J: Carinal resection and reconstruction in thoracic malignancies. J Surg Oncol 110: 239-244, 2014.

4. Fiorelli A, Caterino U, Raucci A and Santini M: A conica self-expanding metallic stent for the management of critical complex tracheobronchial malignant stenosis. Interact Cardiovasc Thorac Surg 24: 293-295, 2017.

5. Takeda T, Itano H, Fukita S, Saitoh M and Takeda S: Bilateral self-expandable metallic stents for lung cancer involving the carina. Respirol Case Rep 1: 48-51, 2013.

6. Sehgal IS, Dhooria S, Madan K, Pattabhiraman V, Mehta R, Goyal R, Akkaraju J and Agarwal R: Placement of tracheobronchial silicone Y-stents: Multicenter experience and systematic review of the literature. Lung India 34: 311-317, 2017.

7. Dutau H, Toutblanc B, Lamb C and Seijo L: Use of the dumon Y-stent in the management of malignant disease involving the carina: A retrospective review of 86 patients. Chest 126: 951-958, 2004.

8. Yang RM, Han XW, Wu G, Li YD and Li FB: Implantation of a self-expandable metallic inverted Y-stent to treat tracheobronchial stenosis in the carinal region: Initial clinical experience. Clin Radiol 62: 1223-1228, 2007.

9. Ayub A, Al-Ayoubi AM and Bhora FY: Stents for airway strictures: Selection and results. J Thorac Dis 9 (Suppl 2): S116-S121, 2017.

10. Righini C, Aniwidyaningsih W, Ferretti G, Pra Y, Raymond CS, Ferretti K, Hustache C, Diab S, Reyt E and Pison CM: Computed tomography measurements for airway stent insertion in malignant airway obstruction. J Bronchology Interv Pulmonol 17: 22-28, 2010.

11. Williams JM, Krebs IA, Riedesel EA and Zhao Q: Comparison of fluoroscopy and computed tomography for tracheal lumen diameter measurement and determination of intraluminal stent size in healthy dogs. Vet Radiol Ultrasound 57: 269-275, 2016.

12. Ernst A, Feller-Kopman D, Becker HD and Mehta AC: Central airway obstruction. Am J Respir Crit Care Med 169: 1278-1297, 2004.

13. Chen H, Zhang J, Qiu XJ, Wang J, Pei YH and Wang YL: Interventional bronchoscopic therapy in adult patients with tracheobronchial mucoepidermoid carcinoma. Chin Med J (Engl) 130: 2453-2458, 2017.

14. Yamamoto K, Kosaba S, Ikeda T and Tokuyasu H: Evaluation of therapeutic method for malignant tumors involving the tracheal carina. Kyobu Geka 54: 581-584, 2001 (In Japanese).

15. Madan K, Dhooria S, Sehgal IS, Mohan A, Mehta R, Pattabhiraman V, Goyal R and Agarwal R: A multicenter experience with the placement of self-expanding metallic tracheobronchial Y stents. J Bronchology Interv Pulmonol 23: 29-38, 2016.

16. Qiao Y, Fu YF, Cheng L, Niu S and Cao C: Placement of integrated self-expanding Y-shaped airway stent in management of carinal stenosis. Radiol Med 121: 744-750, 2016.

17. Mudambi L, Miller R and Eapen GA: Malignant central airway obstruction. J Thorac Dis 9 (Suppl 10): S1087-S1110, 2017.

18. Parissis $\mathrm{H}$ and Young V: Carinal surgery: Experience of a single center and review of the current literature. J Cardiothorac Surg 5: $51,2010$.

19. Yamamoto K, Miyamoto Y, Ohsumi A, Imanishi N and Kojima F: Surgical results of carinal reconstruction: An alterative technique for tumors involving the tracheal carina. Ann Thorac Surg 84: 216-220, 2007
20. Maniwa Y: Surgical treatment of airway disease. J Thorac Dis 8: E78-E82, 2016.

21. Chen CH, Wu BR, Cheng WC, Chen CY, Chen WC, Hsia TC, Liao WC, Tu CY and Hsu WH: Interventional pulmonology for patients with central airway obstruction: An 8-year institutional experience. Medicine (Baltimore) 96: e5612, 2017.

22. Jeong BH, Um SW, Suh GY, Chung MP, Kwon OJ, Kim H and Kim J: Results of interventional bronchoscopy in the management of postoperative tracheobronchial stenosis. J Thorac Cardiovasc Surg 144: 217-222, 2012.

23. Folch E and Keyes C: Airway stents. Ann Cardiothorac Surg 7: 273-283, 2018.

24. Ozdemir C, Sokucu SN, Karasulu L, Onur ST and Dalar L: Placement of self-expandable bifurcated metallic stents without use of fluoroscopic and guidewire guidance to palliate central airway lesions. Multidiscip Respir Med 11: 15, 2016.

25. Conforti S, Durkovic S, Rinaldo A, Gagliardone MP, Montorsi E and Torre M: Self-expanding y stent for the treatment of malignant tracheobronchial stenosis. Retrospective study. Arch Bronconeumol 52: e5-e7, 2016 (In English, Spanish).

26. Fu YF, Wei N, Zhang K and Xu H: Subcarinal ventilation-assisted Y-shaped stent insertion under local anesthesia for patients with complex tracheobronchial stenosis: Initial clinical experience. Diagn Interv Radiol 20: 330-334, 2014.

27. Madan K, Shrestha P, Garg R, Hadda V, Mohan A and Guleria R: Bronchoscopic management of critical central airway obstruction by thyroid cancer: Combination airway stenting using tracheal and inverted-Y carinal self-expanding metallic stents. Lung India 34: 202-205, 2017.

28. Fortin M, MacEachern P, Hergott CA, Chee A, Dumoulin E and Tremblay A: Self-expandable metallic stents in nonmalignant large airway disease. Can Respir J 22: 235-236, 2015.

29. Zhu JH, Lei M, Chen EG, Qiao Q and Zhong TD: Ventilation strategy and anesthesia management in patients with severe tracheal stenosis undergoing urgent tracheal stenting. Acta Anaesthesiol Scand 62: 600-607, 2018.

30. Fadaizadeh L, Hosseini MS and Dabir S: Role of laryngeal mask airway in interventional bronchoscopy procedures for upper tracheal stenosis: Case series. Middle East J Anaesthesiol 22: 223-227, 2013.

31. Fernandez-Bustamante A, Ibanez V, Alfaro JJ, de Miguel E, Germán MJ, Mayo A, Jimeno A, Pérez-Cerdá F and Escribano PM: High-frequency jet ventilation in interventional bronchoscopy: Factors with predictive value on high-frequency jet ventilation complications. J Clin Anesth 18: 349-356, 2006.

32. Mieda H, Nagano Y, Iwasaki E, Oishi Y, Sasai T, Shin Y, Watanabe Y, Oku S, Fukushima T and Tokioka H: Two cases of airway stent placement to treat tracheal and bronchial fistula using general anesthesia under spontaneous respiration. Masui 61: 880-884, 2012 (In Japanese).

33. Tanigawa N, Kariya S, Komemushi A, Nakatani M, Yagi R and Sawada S: Metallic stent placement for malignant airway stenosis. Minim Invasive Ther Allied Technol 21: 108-112, 2012.

34. Wang Y, Guo JH, Zhu GY, Zhu HD, Chen L, Lu J, Wang C and Teng GJ: A novel self-expandable, radioactive airway stent loaded with (125)I seeds: A feasibility and safety study in healthy beagle dog. Cardiovasc Intervent Radiol 40: 1086-1093, 2017.

35. Doyle DJ, Abdelmalak B, Machuzak M and Gildea TR: Anesthesia and airway management for removing pulmonary self-expanding metallic stents. J Clin Anesth 21: 529-532, 2009.

36. Alazemi S, Lunn W, Majid A, Berkowitz D, Michaud G, Feller-Kopman D, Herth F and Ernst A: Outcomes, health-care resources use, and costs of endoscopic removal of metallic airway stents. Chest 138: 350-356, 2010

37. Bhandari A, Wang YH, Lv SX, Xia EJ and Wang OC: Novel strategy of stents in thyroid mass: A case series report of managing severely dyspneic patients. Onco Targets Ther 10: 4997-5004, 2017.

38. Wang H, Zhou Y, Yamaguchi E, Zhou Y, Li D, Zou H, Luo L and $\mathrm{Ma} \mathrm{H}$ : Endoscopic removal of metallic airway stents. J Bronchology Interv Pulmonol 18: 31-37, 2011.

39. Schmidt B, Massenkeil G, John M, Arnold R and Witt C: Temporary tracheobronchial stenting in malignant lymphoma. Ann Thorac Surg 67: 1448-1450, 1999. 